\title{
La formación de profesores de ciencias a través de su interacción en Comunidades de Desarrollo Profesional
}

\author{
Álvaro García Martínez \\ Profesor del proyecto Curricular de Licenciatura en Química. Facultad de ciencias y Educación. \\ Universidad Distrital Francisco José de Caldas. alvaro.garcia@udistrital.edu.co
}

Recibido: 03-09-2009

\section{Resumen}

La formación del profesorado se ha venido trabajando desde diferentes perspectivas, en este documento se plantea una propuesta para abordarla desde de la creación de comunidades que tienen como objeto el desarrollo profesional de sus participantes. El modelo se desarrolla en cinco fases que intentan modificar los ámbitos de formación de los profesores para que poco a poco vayan actuando como diseñadores críticos y reflexivos. Este modelo se fundamenta en el uso del diseño curricular como una vía fundamental para lograr el desarrollo profesional de los profesores durante su interacción en la comunidad. Como caso particular se presenta una experiencia de implementación del proceso de formación adelantada con profesores a nivel universitario quienes trabajan en el área de química.

\begin{abstract}
Traditional teacher training has been worked since many perspectives, in this paper we pretend to broach it since the consolidation of communities which object is the professional development of their members. The model is developed in five phases, where each one of them tries to modify the fields of the teachers' education becoming them into critic and thoughtful designers. This model is supported on the use of the curricular designing as the core to achieve the professional development of teachers during their interaction with the community. As a particular case, we show the implementation of the process followed by professors who work at the chemistry area.
\end{abstract}

\section{Introducción}

La formación del profesorado ha ocupado un espacio de gran importancia en las investigaciones que se adelantan en Didáctica de las Ciencias, esta se ha abordado tanto a nivel inicial como en ejercicio y en diferentes niveles de formación, profesores de primaria, secundaria y universitarios. Se han desarrollado estudios sobre diferentes aspectos de la formación docente, sus ideas y creencias desde la naturaleza de la ciencia, sobre la enseñanza, sobre el aprendizaje, sus prácticas docentes, etc. También se ha estudiado la influencia de las emociones, la identidad y lo humano del trabajo docente en su desempeño profesional (O'Connor, 2008), los cuales son contemplados por estándares profesionales para evaluar el desempeño del profesor en algunos países como Australia. O'Connor desarrolla un estudio en donde pone de manifiesto la gran influencia que tienen las emociones y el carácter humano en el ejercicio docente y como estos elementos se evidencian en dimensiones de tipo profesional, de desempeño y de tipo filosófico, e insiste en la importancia de tenerlas en cuenta a la hora de hablar de "Estándares Profesionales para la Enseñanza".

Ciertos proyectos de desarrollo profesional tienen en común el apoyo que brindan a los profesores para examinar y cambiar tanto sus creencias y su conocimiento como su práctica docente. Consideran lo suficientemente importantes las creencias y el conocimiento que poseen los 
profesores como para someterlos a un examen detenido y a una posible transformación. De otro lado, ponen de relieve la enseñanza de una materia docente concreta en lugar de centrarse en enfoques generalistas o universales sobre la enseñanza. Lo relevante es que, con un trabajo sistemático e intenso, los profesores pueden llevar a cabo cambios sustanciales en su conocimiento, sus creencias y sus prácticas, cambios que tienen unas orientaciones compatibles con una visión que apuesta por la reforma de la enseñanza y el aprendizaje (Putnam y Borko, 1997).

A pesar de los estudios realizados, cuando se habla de la formación del profesor de ciencias que se encuentra en ejercicio, surgen muchos interrogantes, en tomo a ¿Cuáles serían los fines de dicha formación?, ¿Cómo debe realizarse?, ¿Qué se debe trabajar con los docentes?, ¿Debe centrarse en una formación en el cómo enseñar o induir elementos de la disciplina misma?, en fin... realizar toda una planeación curricular para la estructuración de un programa de formación y actualización docente.

Analizando la coherencia de los objetivos formativos con las demandas que día a día se le presentan al profesorado para que llegue a ser considerado como un profesional experto, se pueden plantear cuatro grandes metas, en auanto a los programas de formación se refiere (Valcacer y Sánchez, 2000):

> Mejorar el conocimiento de los profesores en relación con la asignatura que enseñan, sobre todo mediante un aumento del conocimiento del contenido de enseñanza y del conocimiento didáctico del contenido.

$>$ Cambiar las concepciones y prácticas docentes de los profesores hacia enfoques coherentes con presupuestos constructivistas.

> Formar al profesor como diseñador de proyectos curriculares e investigador de su actuación docente en el aula.

$>$ Desarrollar actitudes y prácticas docentes más colaborativas, críticas y autónomas.

Alrededor de estas metas se han adelantado diversidad de trabajos de investigación que intentan generar mejores propuestas sobre cómo formar a los profesores de ciencias, para nuestro caso nos hemos orientado por la creación de comunidades que trabajan en el diseño curricular para potenciar su desarrollo profesional docente.

\section{Desarrollo}

El área de formación del profesorado ha venido evolucionando en la formulación de orientaciones teóricas y metodológicas, por lo aual la perspectiva en los programas ha venido cambiando y con ellos la terminología, por decir, términos como Teacher Education o Teacher Training han venido desapareciendo por las implicaciones que acarrea su uso, y algunos autores lo emplean aún pero para evitar ser ubicados en la imagen dásica reduccionista hacen las respectivas adaraciones y lo que entienden por este, últimamente se emplea el término desarrollo del profesor o desarrollo profesional del profesorado (Teacher Development o Teacher Professional Development).

La formación del profesorado no hay que plantearla como un "cambio", sino más bien como un proceso interno de "crecimiento" y de "desarrollo" gradual a partir de lo que ya piensan y hacen, de los problemas reales de enseñanza y aprendizaje de las ciencias, de las preocupaciones cotidianas del profesor, potenciando y apoyando la motivación, la disponibilidad, la colaboración y el compromiso de los profesores en su propio desarrollo profesional (Mellado, 2003).

Nosotros asumimos el desanrollo profesional del profesorado como una serie de procesos sucesivos de autorregulación metacognitiva que llevan a un crecimiento en los ámbitos que orientan la profesión docente (ámbito personal, de conocimientos y estructuras interpretativas, y el 
ámbito práctico, todos ellos en el mundo personal del profesor) producto de la comprensión, puesta en práctica y de la relación entre lo que piensan, sienten, y hacen en su aula y en su institución; dichos ámbitos se ven influenciados permanentemente por el ámbito externo (Garća, 2009).

Este desarrollo profesional implica que los profesores y profesoras asuman que pueden aprender desde la diversidad, desde lo social, desde el contexto, desde la actividad intelectual compartida y siendo conscientes de su proceso de aprendizaje personal. Cochran-Smith (1998) plantea que el desarrollo profesional de los profesores es "una construcción de cultura" no es un entrenamiento de habilidades; investigaciones sobre el trabajo de los profesores y sus sitios de trabajo sugieren que las mejores prácticas de formación del profesorado son aquellas que proporcionan oportunidades para los profesores para identificar, reconsiderar, soportar, o modificar creencias sobre el salón de dase y prácticas que soporten o profundicen en las oportunidades de aprendizaje de los estudiantes y en oportunidades para la vida.

Trabajar desde una perspectiva colaborativa e interdisciplinaria probablemente ha sido uno de los retos más grandes que se han visto. No es fácil generar un clima colaborativo dentro de unas culturas cuyas características típicas son el trabajo individualizado y anquilosado que desarrollan en la universidad. Es difícil crear un escenario común en el cual los profesores son capaces de trabajar juntos si persisten las diferencias en sus puntos de vista sobre la enseñanza. Dejar de lado el estudio de los contenidos y centrarse más en el trabajo en equipos y en las reflexiones sobre la práctica, generan un primer paso para estudiar las prácticas de enseñanza en la universidad.

Para que los profesores puedan desenvolverse de forma óptima en estos contextos, en muchos casos requerirá importantes cambios en su conocimiento, sus creencias y su práctica. Esto implica que se generen propuestas de formación del profesorado que los oriente hacia una actuación más acorde con los retos de este nuevo milenio, lo aul implica que se planteen programas de formación que se basen en experiencias exitosas en formación del profesorado que tengan presente elementos importantes como los mencionados por Putnam y Borko (1997):

$>$ Los profesores deberían ser tratados como personas que están aprendiendo activamente y que construyen sus propias interpretaciones;

Debería reconocerse el poder de los profesores y deberían ser tratados como profesionales;

$>$ La formación del profesorado debe centrarse en la práctica del aula;

> Los profesores de formación del profesorado deberían tratar a los maestros de la misma manera como esperan que los maestros traten a sus alumnos.

Putnam y Borko (1997) han denominado a estos tópicos como mantras, ya que quieren reconocer tanto la importancia de las ideas que subyacen a estas afirmaciones como la precaución que hay que tener para no simplificarlas en demasía y hacer de ellas un uso indiscriminado.

En relación con las comunidades de aprendizaje este término ha venido usándose cada vez con mayor frecuencia, se habla de comunidades de trabajo, comunidades colaborativas, al punto de referirse a comunidad virtual, donde se puede pagar por un servicio y para que tenga acceso a él se debe pagar, de tal modo que se le asigna un nombre de usuario y una dave de acceso, imágenes tradicionales de las que nos alejamos en lo que hemos desarrollado.

Otra visión es la de Resnik (1991) quien habla de comunidades de discurso como aquellas comunidades que comparten formas de pensamiento y comunicación a través de la interacción continua, es decir que los individuos han llegado a compartir formas comunes de pensar y expresar sus ideas. En esta línea de pensamiento surge el aprendizaje en comunidades, el cual, se interpreta como aquel conjunto amplio y heterogéneo de metodologías de enseñanza organizadas y estructuradas que orientan las actividades de los individuos cuando trabajan juntos, en grupo o en equipos, en situaciones que los convocan para lograr un propósito determinado. 
Al hablar de comunidades de profesionales se observa que existe cierta cantidad de literatura sobre la temática, pero al buscar sobre comunidades de profesores es mucho menor y hay mucho por hacer. Se han estudiado comunidades de otras profesiones como medicina o leyes, pero al refiexionar un poco sobre una comunidad de profesores, se observa que a diferencia de otras, es muy poco lo que el profesor puede decidir sobre su propia comunidad, ya que la mayoría de lo que él trabaja cotidianamente se hace por mandatos de niveles superiores como los constructores de políticas educativas y de personas que están lejos de la realidad de las aulas de dase de hoy. Comparada con medicina o leyes, la educación no tiene la posibilidad de crear un lenguaje compartido de normas y valores.

En el trabajo de comunidades de profesores se destaca su carácter local, donde las interacciones cara a cara, el diálogo constructivo, y la confianza son elementos indispensables para la construcción de la cohesión en el grupo. Una definición interesante de comunidad es la que presentan Bellah et. al. (1985), la cual la desciben como:

"un grupo de personas que son socialmente interdependientes, quienes participan entre sí en discusiones y tomando decisiones, y quienes comparten ciertas prácticas que definen la comunidad y son nutridas por esta"

De allí se desprende que es un proceso que es lento y a largo plazo, ya que los individuos en sus diálogos e interacciones van construyendo historia como grupo, lo aual les va dando identidad al ir compartiendo cada vez más elementos y construyendo propuestas a partir del trabajo en equipo.

Un aspecto dave de las comunidades de profesores es que estos crean espacios de aprendizaje de los profesores de forma continua (Cochran-Smith, 1998). Sin embargo la gran cantidad de asignación de cursos a los profesores, en el caso de educación media, el número de estudiantes en los cursos y el total por día es muy alto, lo que hace que los profesores no tengan ningún espacio para trabajar en equipo, con lo cual el único espacio que les queda es el del descanso escolar en su jomada de trabajo que alcanza solo para comer algo. En el caso de la educación superior varia un poco ya que al hacer este proceso como algo formal, se pueden generar los intervalos de tiempo necesarios.

Las discusiones sobre la práctica son de gran importancia, en la medida en que los profesores pueden hablar sobre casos concretos y presentar diferentes puntos de vista sobre los mismos, generando un ambiente de reflexión crítica permanente de tipo metacognitivo. Cuando se participa en estos seminarios, los profesores están aprendiendo nuevas formas de aprendizaje sobre su práctica docente y al mismo tiempo están construyendo nuevas formas de discurso para hablar de su trabajo docente.

Tomando como referencia lo mencionado, interpretamos a la comunidad de desarrollo profesional de profesores, CODEP, como

un grupo de profesores que se reconocen como profesionales de la educación, los cuales partiajpan en discusiones coíticas y reflexivas para la toma de decisiones en tomo a su objeto de estudio, la enseñanza y el aprendizaje en su aula (e institución) y otros procesos que allí se desarrollan y que las condicionan. Esta comunidad comparte poco a poco ciertas prácticas y referentes que se van constuyendo como producto de esta dinámica, que la definen y retroalimentan.

Cuando se habla que los profesores se reconozcan como profesionales, hace referencia al reconocimiento que hacen ellos mismos como profesores universitarios, que su objeto de estudio es 
la enseñanza de la química (que va relacionado al conocer cómo aprenden sus estudiantes) y todas las implicaciones de ésta en su aula y su institución; lo aul se fue logrando poco a poco, y a medida en que se iba consolidando la comunidad.

La comunidad de desarrollo profesional de profesores que se describe a continuación se diseñó a partir de los referentes descritos y se implementó con profesores universitarios del área de química, durante dos años ${ }^{1}$. Se tomó como eje de estudio y reflexión con los profesores universitarios el uso de la historia de la química para el mejoramiento de la docencia universitaria en los casos particulares de la química general y la fisicoquímica. La CODEP se ha soportado en referentes particulares de los programas de su dase, pero al mismo tiempo presenta elementos propios que lo diferencian de otras propuestas, los cuales se describen a continuación. En primer lugar, cuando se realizó el programa de formación se trabajó con los profesores el cómo enseñar unos contenidos estudiados y reflexionados a la luz de la historia de la química, los cuales fueron analizados y construidos bajo los principios de comunidad de aprendizaje a nivel profesional; y surge aquí el segundo aspecto, el contribuir a la formación de un profesional que vea la necesidad latente de trabajar en equipo, de socializar sus conocimientos, sus aprendizajes y obviamente sus problemas a la hora de llevarlo a la acción. Finalmente, estos elementos contribuyen a una perspectiva que busca el desarrollo del profesor como un profesional, el cual se interroga y formula problemas de investigación de su práctica docente, diseña y lleva al aula propuestas de innovación que buscan superar problemas y poner a prueba hipótesis de trabajo consensuadas en equipo, construyendo y reconstruyendo nuevamente conocimiento didáctico de su disciplina de formación básica.

Los profesores universitarios se vincularon al programa de manera voluntaria y con la intención de mejorar su docencia. El carácter voluntario es un elemento fundamental que permite un alto grado de pertenencia con la comunidad, sin embargo no implica que todos tengan la misma responsabilidad con la comunidad ni con sus actividades, pero ayuda muchísimo para el desarrollo de las tareas propuestas y el logro de la metas fijadas.

El coordinador de la CODEP, y autor de este documento, tuvo que desarrollar varios roles, entre ellos de negociador de intereses, es decir, que los objetivos de la investigación no se diluyeran y por otro lado responder a las expectativas de los miembros de la comunidad. Un segundo rol, de líder académico el cual irradio confianza en el grupo de tal forma que ellos veían que no es algo para perder el tiempo y que no tendrá continuidad en el tiempo; en tercer lugar como persona, que sabía escuchar, que valoraba lo que los otros miembros dicen y hacen y que los motivaba y presionaba cuando era necesario, pero lo más importante es que les haga sentir su papel protagónico de constructores de conocimiento didáctico; y finalmente de compañero que aporta, que cuestiona y que presenta altemativas. Al respecto se plantea que, frente al papel de la persona que orienta el proceso de formación del profesorado, ésta debe tener ciertas características que le permitan generar un ambiente propicio para el desarrollo de las actividades previstas; así, Richardson (1992) afirma que, para ser eficaz, el responsable del desarrollo del profesorado en un contexto de este tipo debe tener un sólido conocimiento sobre la investigación, porque la presentación de este conocimiento es más útil cuando surge como respuesta a las auestiones suscitadas en el curso de la conversación. El responsable del desarrollo del profesorado también ha de tener cuidado en ser humilde, incluso modesto, al presentar la información y los puntos de vista para así poder enfrentarse a la norma dominante en las escuelas de considerar que los responsables del desarrollo del profesorado vinculados a la universidad son los "expertos".

La CODEP se les planteó a los profesores como un intento por contribuir al mejoramiento de la docencia universitaria de los participantes, y su orientación en este caso particular, ha sido tener en

\footnotetext{
${ }^{1}$ Los profesores universitarios tienen tútulo de químicos e ingenieros químicos, con maestrías y doctorado en
} química. 
auenta las relaciones entre la historia de la química, la filosofía de las ciencias y la didáctica de la química, como elementos que fundamentan las acciones docentes. Una vez ellos estuvieron de acuerdo con el objetivo, se organizó un plan de trabajo en conjunto que les dio la posibilidad de desarrollar sus propias ideas y aportar desde su experiencia personal, pero sin descuidar el objetivo del proceso que se había previsto, es decir, los contenidos básicos del programa.

Los aspectos fundamentales para que se pueda llevar a cabo la agenda de una comunidad son el tiempo y el espacio, lo cual se consiguió en la medida en que se logró una descarga académica de dos horas a la semana para los profesores participantes del proyecto y se ubicó en el horario de cada uno de ellos para que les coincidiera un día a la semana para trabajar en la comunidad; este tiempo se prolongó en algunos casos, ya que ellos fueron moviendo sus actividades antes o después de la franja, dependiendo de las necesidades y de sus posibilidades de horario y de trabajo. En cuanto al espacio, las actividades se desarrollaron en una oficina de un grupo de investigación el aual dirige el autor y se contó con los laboratorios de química para realizar los trabajos experimentales necesarios. En este sentido, Grossman y Wineburg (2000) manifiestan que es indispensable, al hablar de una comunidad profesional, que el lugar de reuniones sea la misma institución y que esté en la jornada de trabajo de los profesores, ofreciendo la posibilidad de generar una transformación individual al mismo tiempo que una transformación en la organización social en donde los individuos trabajen. Estos aspectos son complejos para los casos de primaria y secundaria por la cantidad de horas semanales de trabajo que se tienen y el número de estudiantes.

Para el desarrollo de la CODEP se han programado cinco fases que se mencionan a continuación:

Fase 1. Identificación de propósitos y selección de contenidos.

Fase 2 Selección del tópico y área de la historia de la ciencia a estudiar.

Fase 3. Diseño de la herramienta de enseñanza/aprendizaje.

Fase 4. Implementación y análisis del proceso de aplicación de la herramienta.

Fase 5. Reflexión metacognitiva sobre el proceso desarrollado.

En esta primera fase de la CODEP se pretende, en primera instancia, identificar las ideas del profesorado sobre tópicos que se consideran de gran relevancia para el desarrollo del programa, los aules pueden ser aspectos que condicionen, proyecten y sean fundamentales para el desarrollo profesional del profesorado.

Retomando el diagnóstico anterior, y continuando con el trabajo de comunidades, se inicia la segunda fase en la cual se determina el tópico que se podńa y sería pertinente abordar para estudiar. De esta manera se selecciona el tipo de orientación desde la historia de las ciencias, que sería más interesante y pertinente trabajar con los profesores, entre las auales pueden estar: ciencia y cultura, comunicación en ciencia, género, instrumentos y prácticas experimentales, aradernos de notas, etc.

En la tercera fase se pretende evidenciar la manera como interpretan los profesores el uso de la historia de las ciencias en un contexto escolar y qué análisis realizan para su posterior implementación. De igual manera permite reflexionar sobre la manera en que ellos conciben el mejoramiento de su enseñanza, promoviendo el desarrollo de aprendizajes significativos, mediante el empleo de la historia de la ciencia.

En la cuarta fase se contrastan los criterios que planteó el profesor para el diseño de la unidad didáctica y la manera como los retoma al momento de su implementación. Se presta especial interés en la forma en que desarrolla cada una de las actividades y los elementos que resalta, bien

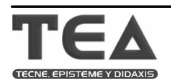


de manera positiva o negativa, con el fin de evidenciar la relación de sus ideas y como éstas orientan su propuesta.

En la última fase se analizan los posibles cambios que se presentaron en los profesores que conforman la CODEP bien en sus actitudes, su lenguaje o sus acciones. Se realiza de igual forma una reflexión metacognitiva sobre las relaciones historia, filosofía y enseñanza de las ciencias.

\section{Conclusiones}

El trabajo en comunidades de desarrollo profesional ubica a los docentes en un papel de aprendices, lo cual facilita la construcción de propuestas a partir del trabajo colectivo, posibilitando la generación de actitudes críticas, que permitió la creación de reflexiones metacognitivas de forma permanente en los participantes.

Las prácticas docentes de los profesores fueron cambiando progresivamente en la medida en que se fueron apropiando de su papel de diseñadores en sus propias asignaturas, producto del trabajo en la comunidad con los otros profesores. Se rediseñaron los cursos completos de cada asignatura a la luz de perspectivas constructivistas de resolución de problemas soportadas en el uso de la historia de la química.

El trabajar en propuestas que impliquen diseño curricular posibilita que se genere todo un proceso de desarrollo profesional de los profesores en la medida en que influye de manera directa en los ámbitos de la profesión docente, lo aual se observó en los profesores participantes, al analizar sus ideas, sus acciones y los productos generados.

\section{Bibliografía}

Bellah, R. N., Madsen, N., Sullivan, W. M., Swidler, A., y Tipton, S. M. (1985). Habits of the heart; Individualism and commitment in American life. Berkeley, CA: University of California Press.

Cochran-Smith, M. (1998). Teaching for social change: Toward a grounded theory of teacher education. En A. Hargreaves, A. Lieberman, M. Fullan \& D. Hopkins, (Eds), The international handbook of educational change. Amsterdam: Kluwer Academic.

García, M. (2009). Aportes de la historia de la ciencia al desarrollo profesional de profesores de química. Tesis Doctoral. Universitat Autonoma de Barcelona.

Grossman, P. Y Wineburg, S. (2000). What makes teacher community different from a gathering of teachers? Centre for the study of teaching and policy. University of Washington.

Mellado, V. (2003). Cambio didáctico del profesorado de ciencias experimentales y filosofía de la ciencia. Enseñanza de las ciencias. 21 (3).

O'Connor, Kate (2008). "You choose to care": Teachers, emotions and professional identity. Teaching and Teacher Education. Vol. 24

Putnam R. T. \& Borko, H. (1997). Teacher learning: Implications of New Views of Cognition. En B. J. Biddle et al (Eds), International Handbook of Teachers and Teaching. Amsterdam: Kuwer Academic.

Resnick (1991). Shared cognition: thinking as social practice. En Resnick, Levine y Teasley (comps), Perspectives on socially shared cognition, Washington, DC American Psychological Association.

Valcacer y Sánchez (2000). La formación del profesorado en ejercicio. En: Perales y Cañal. Didáctica de las ciencias experimentales. Marfil. 